\title{
Quadratic integrand double-hybrid made spin-component-scaled
}

\author{
Éric Brémond, ${ }^{1, a)}$ Marika Savarese, ${ }^{1}$ Juan C. Sancho-García, ${ }^{2}$ Ángel J. Pérez-Jiménez, ${ }^{2}$ \\ and Carlo Adamo ${ }^{1,3,4}$ \\ ${ }^{1}$ CompuNet, Istituto Italiano di Tecnologia, via Morego 30, I-16163 Genoa, Italy \\ ${ }^{2}$ Departamento de Química Física, Universidad de Alicante, E-03080 Alicante, Spain \\ ${ }^{3}$ Chimie ParisTech, PSL Research University, CNRS, Institut de Recherche de Chimie Paris IRCP, \\ F-75005 Paris, France \\ ${ }^{4}$ Institut Universitaire de France, 103 Boulevard Saint Michel, F-75005 Paris, France
}

(Received 3 February 2016; accepted 7 March 2016; published online 22 March 2016)

\begin{abstract}
We propose two analytical expressions aiming to rationalize the spin-component-scaled (SCS) and spin-opposite-scaled (SOS) schemes for double-hybrid exchange-correlation density-functionals. Their performances are extensively tested within the framework of the nonempirical quadratic integrand double-hybrid (QIDH) model on energetic properties included into the very large GMTKN30 benchmark database, and on structural properties of semirigid medium-sized organic compounds. The SOS variant is revealed as a less computationally demanding alternative to reach the accuracy of the original QIDH model without losing any theoretical background. (C) 2016 AIP Publishing LLC. [http://dx.doi.org/10.1063/1.4944465]
\end{abstract}

\section{INTRODUCTION}

Since the pioneering work of Görling and Levy in $1993^{1,2}$ and of Ernzerhof in 1996, ${ }^{3}$ double-hybrid (DH) density-functionals have increasingly become popular within the density-functional theory (DFT) community. ${ }^{4-7}$ They introduce a fraction of nonlocal correlation computed according to the second-order perturbation theory (PT2) into a global-hybrid expression,

$$
E_{x c}^{\mathrm{DH}}=a_{x} E_{x}^{\mathrm{EXX}}+\left(1-a_{x}\right) E_{x}^{\mathrm{DFA}}+\left(1-a_{c}\right) E_{c}^{\mathrm{DFA}}+a_{c} E_{c}^{\mathrm{PT} 2},
$$

where $a_{x}$ and $a_{c}$ govern the fractions of nonlocal exchange and correlation, respectively.

The first three terms (i.e., $E_{x}^{\mathrm{EXX}}, E_{x}^{\mathrm{DFA}}, E_{c}^{\mathrm{DFA}}$ ) denote the exact-like exchange, and the semilocal density-functional approximation (DFA) to exchange and correlation energies, respectively. They are generally computed self-consistently according to the Kohn-Sham procedure even if some alternatives are possible. ${ }^{8}$ The last remaining term (i.e., $E_{c}^{\mathrm{PT} 2}$ ) is computed a posteriori from the converged Kohn-Sham orbitals and can be seen as the sum of the same spin (SS) and opposite spin (OS) electron pair correlation energies such as

$$
E_{c}^{\mathrm{PT} 2}=E_{c}^{\mathrm{OS} \text {, PT2 }}+E_{c}^{\mathrm{SS}, \mathrm{PT} 2} \text {. }
$$

A plethora of investigations already attempted to tune the pair of $\left\{a_{x}, a_{c}\right\}$ parameters entering into Equation (1) via fully empirical ${ }^{8-13}$ or theoretical ${ }^{14-19}$ routes. Following this latter line of design, we recently derived these parameters in a nonempirical fashion from the adiabatic connection model, ${ }^{20}$ using a quadratic polynomial of the coupling integrand parameter and fixing its coefficients through some known limit conditions. ${ }^{14}$ This nonempirical model is named quadratic

\footnotetext{
a)Electronic address: eric.bremond@iit.it
}

integrand double-hybrid (QIDH), and casts $a_{x}=3^{-1 / 3}$ of exact-like exchange and $a_{c}=1 / 3$ of PT2 correlation. ${ }^{14}$ Its performance has already been tested for short- and long-range energetic and structural properties, ${ }^{14,21}$ and its robustness has also been validated with several semilocal DFAs. ${ }^{22}$ Its accuracy is comparable to the most modern densityfunctionals. ${ }^{22}$

Aside of the nonempirical playground, recent works have explored the empirical parameterization route to DHs introducing spin-component-scaled (SCS) variants. ${ }^{23-26}$ These developments were directly inspired from the wavefunction theory, ${ }^{27}$ and more precisely from an original idea of Grimme. ${ }^{28}$ He showed that for low order perturbations (i.e., PT2), the SS component correlates faster than the OS one, i.e., that both components are over- and underestimated, respectively. To compensate such a convergence issue, the SCS scheme weights the PT2 spin electron pairs such as

$$
E_{c}^{\mathrm{SCS}-\mathrm{PT} 2}=a_{s}^{\mathrm{OS}} E_{c}^{\mathrm{OS}, \mathrm{PT} 2}+a_{s}^{\mathrm{SS}} E_{c}^{\mathrm{SS}, \mathrm{PT} 2},
$$

where $a_{s}^{\mathrm{OS}}$ and $a_{s}^{\mathrm{SS}}$ govern the fractions of SS and OS spin electron pair correlation energies, respectively.

The SCS variants of DHs were initially proposed to enhance the performances of the "more conventional" approaches $\left(a_{s}^{\mathrm{SS}}=a_{s}^{\mathrm{OS}}=1\right)$ by an empirical parameterization $\left(a_{s}^{\mathrm{SS}} \neq a_{s}^{\mathrm{OS}}\right)$ on short- and long range-interaction databases. ${ }^{23-26}$ However, while their accuracy largely increases for main-group thermochemistry and kinetic properties with respect to standard DHs, they are not so successful for the accurate treatment of weak interactions due to their incorrect description of the long-range correlation between two nonoverlapping systems. ${ }^{29}$ As a result, some of them include an empirical dispersion correction term ${ }^{30}$ to improve the description of van der Waals interactions.

Still within the wavefunction framework, Head-Gordon and co-workers proposed to radically neglect the SS term 
$\left(a_{s}^{\mathrm{SS}}=0\right)$ and to compensate its weight into the $a_{s}^{\mathrm{OS}}$ parameter. This approach, known as the spin-opposite-scaled (SOS) method, ${ }^{31}$ introduces a considerable advantage by avoiding the computation of exchange-type integrals contained in the SS component, reducing thus the computational effort from $O\left(n^{5}\right)$ ( $n$ referring to the size of the basis set) to $O\left(n^{4}\right)$, computational cost of a global-hybrid, together with the resolution-of-theidentity and a Laplace transformation of the orbital energy denominators. ${ }^{32}$ Some empirical double-hybrids are already taking advantage of this promising SOS scheme. ${ }^{33-35}$

In the present work, we propose a novel SCS variant of DH minimizing the empiricism and we try to intuitively rationalize the SCS and SOS schemes for modern DHs developed within the quantum mechanical framework. To this end, we present a simple and nonempirical expression which recovers the dissociation limit of two weakly bound closed-shell monomers ${ }^{29}$ and we assess its impact on the performance of our recently developed nonempirical QIDH model.

\section{COMPUTATIONAL DETAILS}

All the computations were performed with a development version of the Gaussian suite of programs, ${ }^{36}$ with the exchange-correlation terms evaluated with the semilocal PBE density-functional. ${ }^{37}$ As recommended by Grimme,${ }^{35}$ the large Ahlrichs' type quadruple- $\zeta$ basis set def2-QZVP ${ }^{38}$ is used to compute the GMTKN30 database. ${ }^{35}$ Particularly for electron affinities (G21EA subset), diffuse $s$ and $p$ functions are taken from the Dunning-augmented quadruple- $\zeta$ basis $\operatorname{set}^{39}$ and added to def2-QZVP to obtain the aug-def2-QZVP basis set. More information about the properties included in the GMTKN30 database is given in Table SI and SII of the supplementary material. ${ }^{40}$ Molecular structures included into the B3se47 dataset ${ }^{41}$ were fully optimized with the Dunningaugmented triple- $\zeta$ basis set, and the performances of the DHs were judged according the distance matrix deviations as recently described in Ref. 22.

\section{RESULTS AND DISCUSSION}

At the dissociation limit of two weakly bound closed-shell monomers, the SS and OS spin electron pair parameters should satisfy the relationship, ${ }^{29}$

$$
a_{s}^{\mathrm{OS}}+a_{s}^{\mathrm{SS}}=2 .
$$

Plugging Equation (4) into (3) and imposing the QIDH conditions ${ }^{14}\left(a_{x}=3^{-1 / 3}\right.$ and $\left.a_{c}=1 / 3\right)$ give the one-parameter spin-component-scaled quadratic integrand double-hybrid model,

$$
\begin{aligned}
E_{x c}^{\mathrm{SCS} 1-\mathrm{QIDH}}= & a_{x} E_{x}^{\mathrm{EXX}}+\left(1-a_{x}\right) E_{x}^{\mathrm{DFA}}+\left(1-a_{c}\right) E_{c}^{\mathrm{DFA}} \\
& +a_{c}\left[a_{s} E_{c}^{\mathrm{OS}, \mathrm{PT} 2}+\left(2-a_{s}\right) E_{c}^{\mathrm{SS}, \mathrm{PT} 2}\right],
\end{aligned}
$$

where $a_{s} \in[0,2]$ denotes in the following $a_{s}^{\mathrm{OS}}$. Note that Equation (5) recovers the original QIDH expression (see Eq. (1) with the QIDH conditions) by setting $a_{s}=1$. Next, we use the latter equation to deduce a suitable SOS scheme within the QIDH formalism.

First of all, Figure 1 shows the ratio of both spin electron pair correlation energies for the 114 small closedshell systems included into the G2/148 dataset ${ }^{42-44}$ and with two different Dunning-augmented correlation-consistent basis sets $^{39}$ (denoted here aVnZ, with $n=\mathrm{T}$ or $n=\mathrm{Q}$ ). As general trends, we notice that (i) the SS/OS energetic ratio does not significantly depend on the size of the basis set and (ii) the average over all the closed-shell systems yields to

$$
E_{c}^{\mathrm{SS}, \mathrm{PT} 2} \approx \frac{1}{3} E_{c}^{\mathrm{OS}, \mathrm{PT} 2} \text {. }
$$

Furthermore, most of the compounds have their SS/OS energetic ratio lying within the standard deviation interval $( \pm 0.05)$. Only those strongly prone to dynamical correlation effects (like the silicon-based ones) largely deviate from this rule. Note that a similar ratio was obtained before while computing both same and opposite spin PT2 components from Hartree-Fock orbitals. ${ }^{28}$

Plugging then Equation (6) into (5) gives the one-parameter spin-opposite-scaled quadratic integrand

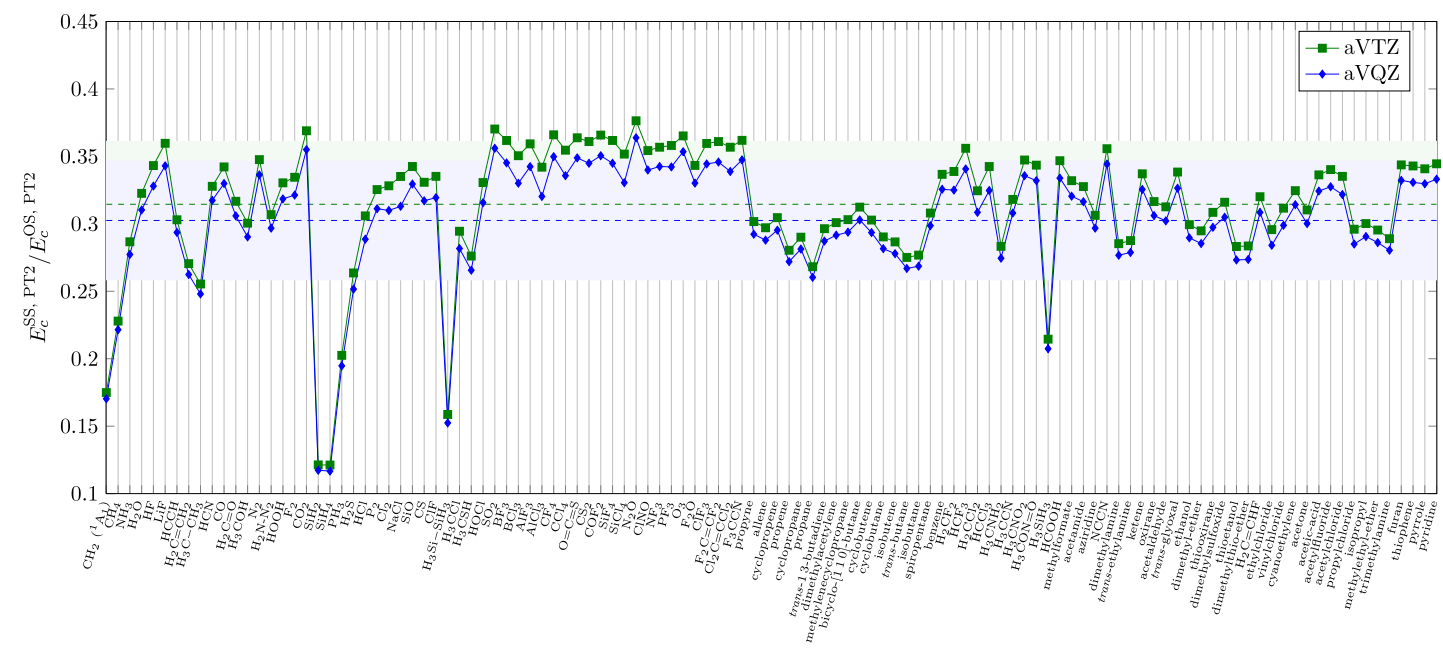

FIG. 1. Ratio of same and opposite spin electronic energies computed with augmented triple and quadruple- $\zeta$ basis sets for 114 closed-shell systems included in the G2/148 atomization energy database. Colored dashed lines denote the statistical averages over all the systems for each basis set, while the colored background represents the standard deviation of the series. 


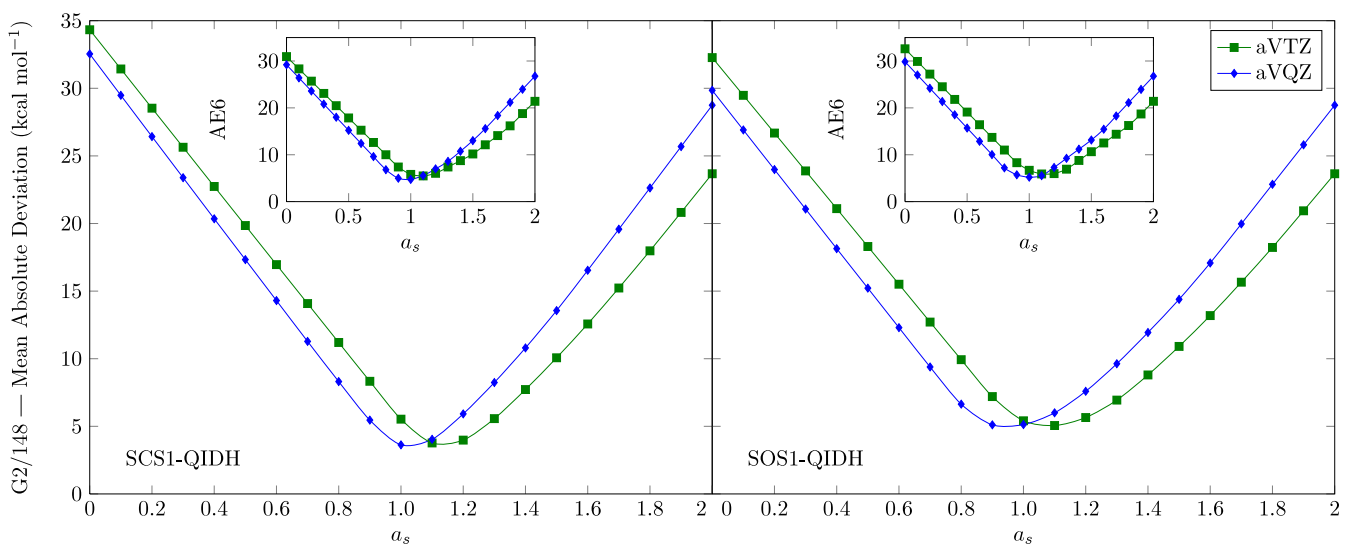

FIG. 2. Mean absolute deviations (in kcal mol${ }^{-1}$ ) for the SCS1-QIDH (left) and SOS1-QIDH (right) models computed on the G2/148 and AE6 (insets) atomization energy databases for augmented triple and quadruple- $\zeta$ basis sets.

double-hybrid model,

$$
\begin{aligned}
E_{x c}^{\mathrm{SOS} 1-\mathrm{QIDH}}= & a_{x} E_{x}^{\mathrm{EXX}}+\left(1-a_{x}\right) E_{x}^{\mathrm{DFA}}+\left(1-a_{c}\right) E_{c}^{\mathrm{DFA}} \\
& +a_{c} \frac{2}{3}\left(a_{s}+1\right) E_{c}^{\mathrm{OS}, \mathrm{PT} 2} .
\end{aligned}
$$

Both SCS1-QIDH and SOS1-QIDH models satisfy the spin-component-scaled relation expressed by Equation (4). We test now their performances within the short-range regime computing a large set of atomization energies and comparing them with the references included into the small but representative $\mathrm{AE}^{45}$ and the large G2/148 databases. Figure 2 reports the statistical mean absolute deviations (MADs) for the two different considered basis sets when varying $a_{s}$.

Independent of the $a_{s}$ parameter, the SCS1-QIDH and SOS1-QIDH models behave similarly on both atomization energy databases but, as it happens for Møller-Plesset theory too, present a slow basis set convergence (see Fig. S1 in the supplementary material ${ }^{40}$ ). Taking the very large aVQZ basis set as reference, computations with the triple- $\zeta$ basis set show a deviation on the minimum position of $\left|\Delta a_{s}\right|=0.1\left(\left|\Delta a_{s}\right|=0.2\right)$, and on the minimum absolute error of $|\Delta \mathrm{MAD}| \approx 0.1(|\Delta \mathrm{MAD}| \approx 0.1) \mathrm{kcal} \mathrm{mol}^{-1}$ for SCS1-QIDH (SOS1-QIDH). Concerning the reference quadruple- $\zeta$ basis set, the minimum is positioned at $a_{s}$ $=1.0\left(a_{s}=0.9\right)$ with a MAD of $3.6(5.1) \mathrm{kcal} \mathrm{mol}^{-1}$ for the $\mathrm{G} 2 / 148$ database.

Remarkably, it is found that the best estimation of the atomization energy properties is obtained for $a_{s}=1.0$ for the SCS1-QIDH model (largest basis set), i.e., the original QIDH model is the best approach to estimate such a property. Interestingly, a comparison with the DSD-PBEPBE case $^{23}$ (a fully empirical SCS DH trained to minimize the errors for short- and long-range interactions) shows how this model casts a similar fraction of exact-like exchange $(0.74$ versus $\sim 0.69$ for the QIDH model) but infringes Equation (4) $\left(a_{s}^{\mathrm{OS}}=1.0\right.$ and $a_{s}^{\mathrm{SS}}=0.6$ ). A similar analogy can also be done for the SOS1-QIDH model with xDH-PBE0, ${ }^{33}$ a fully empirical nonself-consistent SOS DH trained to minimize the errors for heat of formation properties. xDH-PBE0 casts more exactlike exchange than the QIDH model ( 0.83 versus $\sim 0.69)$, and
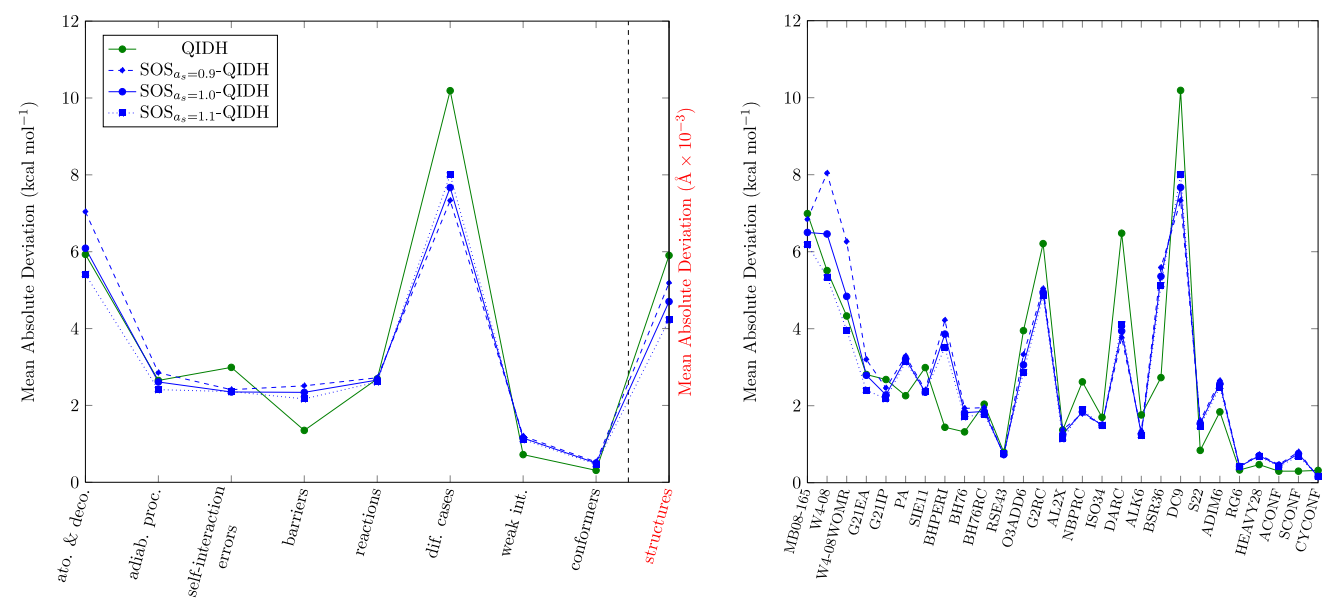

FIG. 3. Mean absolute deviations for a large variety of chemical properties for the original QIDH model and its SOS1-QIDH variant. The left graph shows the performances of the DHs according to the type of properties tested in the GMTKN30 database (in kcal mol ${ }^{-1}$, see Table SI and SII in the supplementary material ${ }^{40}$ ) plus structural properties deriving from the B3se47 dataset (in $\AA$ ). The right graph displays the DH performances on some subsets of the GMTKN30 database (in $\mathrm{kcal} \mathrm{mol}^{-1}$ ). All the reactions are evaluated with the quadruple- $\zeta$ def2-QZVP basis set for energetic properties and with the augmented triple- $\zeta$ aVTZ basis set for structural properties. 
the optimal $a_{s}$ parameter was found equal to 1.4 instead of 1.1 for the SOS1-QIDH model. This difference of 0.3 units is probably due to the excess of exact-like exchange in case of xDH-PBE0 but lies in the same range of $a_{s}$ values.

Generally speaking, while the above tuning of the $a_{s}$ parameter included into the SCS1-QIDH expression converges to the original QIDH model and validates it as the best performing approach, there is still room for improving the performance of the less computationally demanding SOS1-QIDH model. Its performance is now tested on a large variety of short- and long-range energetic properties included into the extended GMTKN30 benchmark set, ${ }^{35}$ and on molecular structure properties considered into the B3se 47 dataset. ${ }^{41}$ Figure 3 reports the MADs for these series of properties for the QIDH and SOS1-QIDH models for three different values of the $a_{s}$ parameter sampled around the minimum of the blue curve represented in Figure 2 (see Table SII in the supplementary material for numerical details $\mathrm{s}^{40}$ ).

Independent of the $a_{s}$ parameter, the SOS1-QIDH variant is rarely worse but often better than or equivalent to the QIDH model, especially for the self-interaction error (SIE) subset, the nine difficult cases included into the DC9 subset, and the determination of structural parameters. Only barrier heights and weak interaction energies are worsened by 1.0 and $0.4 \mathrm{kcal} \mathrm{mol}^{-1}$ respectively, when going from the standard DH to its SOS1-QIDH variant. On one hand, the deletion of the SS component is probably the main cause of the overestimation of energy barrier heights through a general destabilization of the transition-state. On the other hand, the small underestimation of the binding energy of weakly interacting dimers might reflect the lack of nonlocal correlation.

Globally, the effect of the $a_{s}$ parameter on the performance of the SOS1-QIDH model is considered of marginal importance. It mainly plays a role for atomization and decomposition processes, and for the determination of covalent structural parameters, i.e., for properties governed by short-range interactions. On the opposite, long-range interactions are less sensitive to small variations of this parameter. Considering all the tested properties, $a_{s}=1.0$ seems to be a good compromise with respect to the original QIDH model. As a result, the SOS1-QIDH model is defined according to Equation (7) and the following parameters $\left\{a_{x}=3^{-1 / 3}, a_{c}=1 / 3, a_{s}=1.0\right\}$. It leads to the same weight of the OS electron pair component $\left(4 / 3 a_{c} \approx 1.3 a_{c}\right)$ as reported for SOS-MP $2,{ }^{31}$ keeping in mind that $a_{c}=1$ in the latter case.

\section{CONCLUSION}

In this work, we intuitively rationalized the SCS and SOS schemes to DH density-functionals by minimizing the empirical parameterization, and we adapted them to the QIDH model. For that we derived two analytical expressions, called SCS1-QIDH and SOS1-QIDH, ensuring the correct physical description of the long-range correlation between two nonoverlapping systems. Both models are function of only one parameter which can be numerically tuned to recover satisfying performances in the short-range regime. Especially, we showed that the performance of the SCS1-QIDH model converges to the one of the original QIDH models, but that its
SOS1-QIDH variant is an excellent compromise to improve them at a cheaper computational prize. We hope that this work will stimulate the development of novel DH models jointly minimizing the empirical parameterization and reducing the computational effort.

\section{ACKNOWLEDGMENTS}

A.J.P.J. and J.C.S.G. thank the "Ministerio de Economía y Competitividad" of Spain and the "European Regional Development Fund" through Project No. CTQ2014-55073-P for financial support.

${ }^{1}$ A. Görling and M. Levy, Phys. Rev. B 47, 13105 (1993).
${ }^{2}$ A. Görling and M. Levy, Phys. Rev. A 50, 196 (1994).
${ }^{3}$ M. Ernzerhof, Chem. Phys. Lett. 263, 499 (1996).
${ }^{4}$ L. Goerigk and S. Grimme, Wiley Interdiscip. Rev.: Comput. Mol. Sci. 4,
576 (2014).
${ }^{5}$ J. C. Sancho-Garcia and C. Adamo, Phys. Chem. Chem. Phys. 15, 14581
(2013).
6I. Y. Zhang and X. Xu, Int. Rev. Phys. Chem. 30, 115 (2011).
${ }^{7}$ Y. Zhao, B. J. Lynch, and D. G. Truhlar, J. Phys. Chem. A 108, 4786 (2004).
${ }^{8}$ Y. Zhang, X. Xu, and W. A. Goddard, Proc. Natl. Acad. Sci. U. S. A. 106,
4963 (2009).
${ }^{9}$ J. C. Sancho-García and A. J. Pérez-Jiménez, J. Chem. Phys. 131, 084108 (2009).

${ }^{10}$ A. Tarnopolsky, A. Karton, R. Sertchook, D. Vuzman, and J. M. L. Martin, J. Phys. Chem. A 112, 3 (2008).

${ }^{11}$ A. Karton, A. Tarnopolsky, J.-F. Lamère, G. C. Schatz, and J. M. L. Martin, J. Phys. Chem. A 112, 12868 (2008).

${ }^{12}$ T. Schwabe and S. Grimme, Phys. Chem. Chem. Phys. 8, 4398 (2006).

${ }^{13}$ S. Grimme, J. Chem. Phys. 124, 034108 (2006).

${ }^{14}$ E. Brémond, J. C. Sancho-García, A. J. Pérez-Jiménez, and C. Adamo, J. Chem. Phys. 141, 031101 (2014).

${ }^{15} \mathrm{M}$. Alipour, Theor. Chem. Acc. 134, 1 (2015).

${ }^{16}$ N. Q. Su and X. Xu, J. Chem. Phys. 140, A512 (2014).

${ }^{17}$ J. Toulouse, K. Sharkas, E. Brémond, and C. Adamo, J. Chem. Phys. 135, 101102 (2011).

${ }^{18}$ E. Brémond and C. Adamo, J. Chem. Phys. 135, 024106 (2011).

${ }^{19}$ K. Sharkas, J. Toulouse, and A. Savin, J. Chem. Phys. 134, 064113 (2011).

${ }^{20}$ J. Harris, Phys. Rev. A 29, 1648 (1984).

${ }^{21}$ E. Brémond, M. Savarese, N. Q. Su, A. J. Pérez-Jiménez, X. Xu, J. C. Sancho-García, and C. Adamo, J. Chem. Theory Comput. 12, 459 (2016).

${ }^{22}$ E. Brémond, M. Savarese, A. J. Pérez-Jiménez, J. C. Sancho-García, and C. Adamo, J. Phys. Chem. Lett. 6, 3540 (2015).

${ }^{23}$ S. Kozuch and J. M. L. Martin, J. Comput. Chem. 34, 2327 (2013).

${ }^{24}$ S. Kozuch and J. M. L. Martin, Phys. Chem. Chem. Phys. 13, 20104 (2011).

${ }^{25}$ B. Chan and L. Radom, J. Chem. Theory Comput. 7, 2852 (2011).

${ }^{26}$ S. Kozuch, D. Gruzman, and J. M. L. Martin, J. Phys. Chem. C 114, 20801 (2010).

${ }^{27}$ S. Grimme, L. Goerigk, and R. F. Fink, Wiley Interdiscip. Rev.: Comput. Mol. Sci. 2, 886 (2012).

${ }^{28}$ S. Grimme, J. Chem. Phys. 118, 9095 (2003).

${ }^{29}$ R. C. Lochan, Y. Jung, and M. Head-Gordon, J. Phys. Chem. A 109, 7598 (2005).

${ }^{30}$ S. Grimme, J. Antony, S. Ehrlich, and H. Krieg, J. Chem. Phys. 132, 154104 (2010).

${ }^{31}$ Y. Jung, R. C. Lochan, A. D. Dutoi, and M. Head-Gordon, J. Chem. Phys. 121, 9793 (2004).

${ }^{32}$ Y. Jung, Y. Shao, and M. Head-Gordon, J. Comput. Chem. 28, 1953 (2007).

${ }^{33}$ I. Y. Zhang, N. Q. Su, E. Brémond, C. Adamo, and X. Xu, J. Chem. Phys. 136, 174103 (2012).

${ }^{34}$ I. Y. Zhang, X. Xu, Y. Jung, and W. A. Goddard, Proc. Natl. Acad. Sci. U. S. A. 108, 19896 (2011).

${ }^{35}$ L. Goerigk and S. Grimme, J. Chem. Theory Comput. 7, 291 (2011).

${ }^{36}$ M. J. Frisch, G. W. Trucks, H. B. Schlegel, G. E. Scuseria, M. A. Robb, J. R. Cheeseman, G. Scalmani, V. Barone, B. Mennucci, G. A. Petersson, H. Nakatsuji, M. Caricato, X. Li, H. P. Hratchian, A. F. Izmaylov, J. Bloino, G. Zheng, J. L. Sonnenberg, M. Hada, M. Ehara, K. Toyota, R. Fukuda, J. Hasegawa, M. Ishida, T. Nakajima, Y. Honda, O. Kitao, H. Nakai, T. Vreven, 
J. A. Montgomery, Jr., J. E. Peralta, F. Ogliaro, M. Bearpark, J. J. Heyd, E. Brothers, K. N. Kudin, V. N. Staroverov, R. Kobayashi, J. Normand, K. Raghavachari, A. Rendell, J. C. Burant, S. S. Iyengar, J. Tomasi, M. Cossi, N. Rega, J. M. Millam, M. Klene, J. E. Knox, J. B. Cross, V. Bakken, C. Adamo, J. Jaramillo, R. Gomperts, R. E. Stratmann, O. Yazyev, A. J. Austin, R. Cammi, C. Pomelli, J. W. Ochterski, R. L. Martin, K. Morokuma, V. G. Zakrzewski, G. A. Voth, P. Salvador, J. J. Dannenberg, S. Dapprich, A. D. Daniels, Ö. Farkas, J. B. Foresman, J. V. Ortiz, J. Cioslowski, and D. J. Fox, Gaussian Development version Revision H.11, Gaussian, Inc., Wallingford, CT, 2010.

${ }^{37}$ J. P. Perdew, K. Burke, and M. Ernzerhof, Phys. Rev. Lett. 77, 3865 (1996).

${ }^{38}$ F. Weigend and R. Ahlrichs, Phys. Chem. Chem. Phys. 7, 3297 (2005).
${ }^{39}$ T. H. Dunning, K. A. Peterson, and D. E. Woon, Encyclopedia of Computational Chemistry (John Wiley \& Sons, Ltd., 2002).

${ }^{40}$ See supplementary material at http://dx.doi.org/10.1063/1.4944465 for details about the GMTKN30 database.

${ }^{41}$ M. Piccardo, E. Penocchio, C. Puzzarini, M. Biczysko, and V. Barone, J. Phys. Chem. A 119, 2058 (2015).

${ }^{42}$ J. A. Pople, M. Head-Gordon, D. J. Fox, K. Raghavachari, and L. A. Curtiss, J. Chem. Phys. 90, 5622 (1989).

${ }^{43}$ L. A. Curtiss, K. Raghavachari, G. W. Trucks, and J. A. Pople, J. Chem. Phys. 94, 7221 (1991).

${ }^{44}$ L. A. Curtiss, K. Raghavachari, P. C. Redfern, and J. A. Pople, J. Chem. Phys. 106, 1063 (1997).

${ }^{45}$ B. J. Lynch and D. G. Truhlar, J. Phys. Chem. A 107, 8996 (2003). 